\title{
Editorial
}

\section{Astronomy and Astrophysics will move to online-only publication at the start of 2016}

\begin{abstract}
Developments in the field of scientific publishing are accelerating. More extensions, facilities, and useful links are constantly being added to articles published in $A \mathcal{E} A$ through the joint efforts of its Editors and Publisher. We need only mention $3 \mathrm{D}$ images, movies, and the e-Pub format as examples. None of these new additions are available in the printed edition of the Journal; for this reason, the online edition of our Journal is the publication of record and has been so for over a decade.
\end{abstract}

As have the governing bodies of other major astronomical journals, the Board of Directors and the Editors of $A \mathcal{E} A$ have therefore been considering the future of the printed edition.

Indeed, the printed edition has become a mere addition to the online edition, available only in connection with the electronic subscription and at additional cost. Our continuing to provide it when more and more of our subscribers choose the online-only edition will soon be no longer economically viable.

At the $A \mathcal{E} A$ Board of Directors meeting in Bern, Switzerland, it was agreed that the Chair of the Board, in consultation with the Executive Committee and the Editors, would take the decision to terminate the printed edition of the Journal. After ascertaining that reliable electronic archives, as provided for example through Portico, are now generally available, and considering that archival versions of the Journal are kept at ESO and the CDS, we reached an agreement with the Publisher, who is contractually obligated to produce a printed edition, to end the printed edition as of January 1, 2016.

What will and will not change?

The electronic edition will be the only edition proposed to subscribers, who will no longer have to pay for the additional printed edition.

The full-resolution electronic edition of the Journal (in PDF format) will be available on the $A \mathcal{E} A$ and the publisher's websites to subscribers who need to print parts of the journal for their own use.

The successful series of special issues that present the results from special (space) missions and projects will be continued. A printed edition in a single bound volume of these special issues will still be available for purchase from the Publisher at cost.

The data presented in articles will continue to be archived through the CDS. This will be enforced by the Editors.

The Journal and its Editors are keen to keep on adding more facilities for producing scientific papers and exploiting their contents for the benefit of the Authors and the astronomical community at large.

Dr. J. Lub

Chairman of the $A \mathcal{E} A$ Board 